\title{
Negative Feedback in Morphogen Gradients
}

\author{
M. Khong and F.Y.M. Wan \\ Department of Mathematics \\ University of California, Irvine, CA 92697-3875.
}

July 19, 2006

\begin{abstract}
In this paper, the effects of a conventional form of negative feedback on receptor synthesis rate as a mechanism to induce robustness of biological development of the Drosophila wing imaginal disc is analyzed through the initial-boundary value problem in a basic partial differential equation model for the extracellular biological development activities. The existence, uniqueness, linear stability and monotonicity of the steady state signaling gradient are established rigorously. Solutions are then obtained for a special case of the steady state problem to show explicitly the effects of the chosen form of negative feedback. It is evident from the results that the principal effect of such a feedback mechanism is to render the signaling bound morphogen gradient more uniformly distributed except for a narrow layer adjacent to the edge of the posterior compartment of the wing disc. While the change in the magnitude of the maximum signaling morphogen concentration near the ligand source may be kept at an acceptable level, the leveling and flattening of the gradient render it less differential in space (except in the boundary layer) and hence more deviating from the desired gradient for the target biological pattern. The conclusion is then shown to apply to the general case with the help of the theoretical results on monotonicity. These results suggest that negative feedback on the receptor synthesis rate of the chosen form is not effective for promoting robustness. In fact, it drives the system somewhat in the opposite direction, a conclusion supported by the simulation results. The findings in turn suggest more useful forms of the negative feedback for mediating receptor synthesis to offset a higher ligand synthesis rate for our types of systems.
\end{abstract}

\section{Introduction}

Morphogens (also known as ligands) are molecular substances (proteins) that bind to selected signaling cell surface receptors (and other kinds of non-signaling molecules 
not considered herein). The concentration gradients of morphogens bound to their associated signaling cell receptors are known to be responsible for differential cell expressions and the patterning of biological tissues during the developmental phase of the biological host. For a number of morphogen families (including $D p p$ in the wing imaginal disc of Drosophila fruit flies), it is well established that a signaling morphogen is produced at a localized source at some synthesis rate $V_{L}$ and transported away from the source by diffusion (and possibly other transport mechanisms not considered explicitly herein). Some of the transported ligand molecules bind with cell receptors along the way forming signaling ligand-receptor complexes (called bound morphogens or signaling morphogens for brevity). Some of the bound morphogens endocytose into the cell interior while others dissociate to free up ligands to be transported further downstream for possible binding with receptors at other locations. The bound morphogens in the cell interior may degrade and dissolve or exocytose back to the extracellular space. At any instance in time, the concentration of bound morphogen complexes generally decreases with distance away from the morphogen source and this concentration gradient triggers differential cell expressions resulting in a cell tissue pattern (see [2], [3], [5], [16] and other references cited in [8]). The time evolution of the basic morphogen activities (diffusion, reversible binding with renewable receptors and degradation) in Drosophila wing imaginal discs toward a relatively steady signaling morphogen gradient for cell expression has been investigated recently by systems of partial differential equations and auxiliary conditions that mathematically characterize these basic activities $[7,9,17]$. By analyzing mathematically the initial-boundary value problems for these models, the effects of various system rate parameters were delineated. In particular, the concentration of bound morphogen complexes was shown to tend respectively to a unique, linearly stable steady state gradient that is monotone decreasing from the localized source to the edge of the imaginal disc (see $[7,9,17]$ ).

While the mathematical models of $[7,9,17]$ as well as those of [10] established the consistency of diffusion as a morphogen transport mechanism with experimental observations on signaling morphogen gradients and cell expression, they do not exhibit the expected robustness of biological development with respect to system parameter changes. For example, doubling the ligand synthesis rate (due to a substantial temperature change, say) was found to result in a substantial change in model response in the magnitude and shape of the concentration gradients [12]. A numerical measure $E$ was introduced in [12] for characterizing robustness, with $E=0$ being perfectly robust and $E \leq 0.2$ taken (somewhat arbitrarily) to be acceptably robustness. Numerically simulated responses to $10^{6}$ different sets of parameter values in the six dimensional parameter space are seen to be non-robust with $E \geq \ln (2) / \ln (5) \gtrsim 0.43$ in all cases. Evidently, additional biological processes are at work and must be included in the model to ensure robustness of the development with respect to changes in system properties.

In [12], negative feedback, an often used mechanism for mediating excessive changes, 
was applied to the receptor synthesis rate $V_{R}$ in the form

$$
V_{R}=V_{\min }+\frac{V_{\max }-V_{\min }}{1+\Gamma[L R]^{n}}
$$

where $V_{\max }$ and $V_{\min }\left(<V_{\max }\right)$ are the maximum and minimum synthesis rates for receptors, $[L R]$ is the total concentration of signaling (bound) morphogens (both in the cell interior and the extracellular space), and $\Gamma$ and $n$ are two constant parameters with the latter generally referred to as the Hill's coefficient [4]. Evidently, we have $V_{R}=V_{\max }$ in the absence of bound morphogens and $V_{R}$ tends to $V_{\min }$ as $[L R]$ tends to infinity. It was expected that at high morphogen synthesis rate giving rise to a high transient concentration of bound morphogens, the feedback mechanism (1) would reduce the receptor synthesis rate to a substantially lower level to result in concentration gradients differing insignificantly from the response to the normal synthesis rate prior to the rate change and thereby ensuring robustness. Rather surprisingly, the results of numerical simulations for $10^{6}$ sets of parameter values for a system with feedback mechanism (1) show no improvement in the robustness in the model response to a doubling of ligand synthesis rate. In fact, more parameter value sets with larger $E$ values are found in the simulation results for the model with negative feedback.

In this paper, we analyze the effects of a negative feedback of the form (1) on the response of the model system. We will establish that similar to the original system investigated in [9], the initial-boundary value problem for the model system with feedback is well-posed. More specifically, we will prove the existence, uniqueness and linear stability of the monotone decreasing steady state signaling gradient. The various proofs for the present system are more intricate than those in [9] for reasons that will become apparent after we have formulated the mathematical problem. We then obtain useful solutions of the problem for the special case of $n=1$ which will provide us insight to the effects of our particular type of negative feedback. It will be seen from the results that the principal effect of such a feedback mechanism is to render the signaling bound morphogen gradient $[L R]$ more uniformly distributed except for a boundary layer adjacent to the edge of the wing imaginal disc. While the change in the magnitude of the maximum bound morphogen concentration near the ligand source may be kept at an acceptable level by our negative feedback, the leveling and flattening of the gradient render the tissue patterning less differential in space and hence deviate more from the target biological patterning.

The conclusions drawn from the solution for the $n=1$ case will then be extended to the original model system with a general Hill's coefficient $(n \geq 1)$. This suggests that negative feedback on the receptor synthesis rate of the form (1) is not effective for inducing robustness. In fact, it drives the system somewhat in the opposite direction, a conclusion supported by the simulation results of [12]. The findings in turn suggest more appropriate forms of the negative feedback for mediating receptor synthesis should be explored for offsetting a higher ligand synthesis rate. The effects of some of these forms of negative feedback on robustness are being examined. 


\section{An Extracellular Formulation with Receptor Syn- thesis}

As in $[8,9]$, we simplify the development of the wing imaginal disc of a Drosophila fly as a one-dimensional phenomenon. In doing so, we ignore variations in the ventraldorsal direction and the apical-basal direction since extensions of the one-dimensional model to account for developments in these other directions are straightforward (see $[11,17,18]$ for example). To investigate the consequences of negative feedback of signaling morphogen concentration on the receptor synthesis rate, we will work with an extracellular formulation similar to [9]. As we have shown in [10, 13], the results for such a model may be re-interpreted as the corresponding results for a model where morphogen-receptor complexes internalize (through endocytosis) before degradation.

To simplify our discussion, we note that the morphogen production zone divides the wing imaginal disc into the anterior compartment and the posterior compartment. We consider in this paper the part of the wing disc extending from the midpoint, $X=-X_{m}$, of the $D p p$ production zone to the edge of the posterior compartment at $X=X_{\max }$ with morphogen produced only in $-X_{\min }<X<0 . \quad$ Let $[L(X, T)]$ be the concentration (in micromoles) of the diffusing morphogen $D p p$ at time $T$ and location $X$. Let $[R(X, T)]$ and $[L R(X, T)]$ be the concentration of unoccupied receptors and morphogen occupied receptors (or bound morphogens), respectively. For the underlying biological processes of the development described in $[8,9]$, we add to Fick's second law for diffusive transport of $D p p\left(\partial[L] / \partial T=D \partial^{2}[L] / \partial X^{2}, D\right.$ being the diffusion coefficient) terms that incorporate the rate of morphogen binding with receptors, $-k_{o n}[L][R]$, and dissociation, $k_{o f f}[L R]$, with $k_{o n}$ and $k_{\text {off }}$ being the binding rate constant and dissociation rate constant, respectively. In living tissues, molecules that bind receptors do not simply stay bound, some will dissociate and others will (endocytose and) degrade [16]. In accounting for the time rate of change of the Dppreceptor complexes, we allow for constitutive degradation of $[L R]$ by introducing a degradation rate term with a rate constant $k_{\text {deg }}$. There is also a separate accounting of the time rate of change of the concentration of unoccupied receptors as they are being synthesized and degrade continuously in time (with a degradation rate constant

$r_{\text {deg }}$ as in [9]). In this way, we obtain the following reaction-diffusion system for the evolution of three concentrations $[L],[L R]$ and $[R]$ :

$$
\begin{aligned}
\frac{\partial[L]}{\partial T} & =D \frac{\partial^{2}[L]}{\partial^{2} X}-k_{o n}[L][R]+k_{o f f}[L R]+V_{L}(X, T) \\
\frac{\partial[L R]}{\partial T} & =k_{o n}[L][R]-\left(k_{o f f}+k_{\operatorname{deg}}\right)[L R] \\
\frac{\partial[R]}{\partial T} & =V_{R}(X, T)-k_{o n}[L][R]+k_{o f f}[L R]-r_{\operatorname{deg}}[R]
\end{aligned}
$$

for $-X_{\min }<X<X_{\max }$ and $T>0$ where $V_{L}(X, T)$ and $V_{R}(X, T)$ are the rate at 
which $D p p$ and receptors are synthesized, respectively.

In $[7,8,10]$, we were interested only in the portion of the wing disc corresponding to $X>0$ where there is no morphogen production (so that $V_{L}(X, T)=0$ for $X>0$ ) with the introduction of $D p p$ into the region $0<X<X_{\max }$ characterized by a point source at the end $X=0$. A model with a finite $D p p$ synthesis region of the form (2) - (4) but without feedback has been investigated in [9] where the relation between that model and point source models was discussed. Here, we add to the finite $D p p$ production region model of [9] negative feedback of the $[L R]$ concentration on the receptor synthesis rate in the form (1) where the Hill's coefficient $n$ and multiplier $\Gamma$ are constants to be specified.

In the absence of morphogens so that $[L R]=0$, we take $V_{R}(X, T)=V_{\max }(X)$ in order to have a steady state receptor concentration for that case. In the limit as $[L R] \longrightarrow \infty$, we require that $V_{R}$ tends to $V_{\min }(X)\left(<V_{\max }(X)\right)$ to allow for the possibility of steady state $[L],[R]$, and $[L R]$ concentrations also.

With $-X_{\min }$ being the midpoint of the $D p p$ production region, we have by symmetry

$$
X=-X_{\min }: \frac{\partial[L]}{\partial X}=0 \quad(T>0)
$$

The far end of the wing disc, i.e., the edge of the posterior chamber, is taken to be a sink so that

$$
X=X_{\max }:[L]=0 \quad(T>0) .
$$

At $T=0$, we have the initial conditions

$$
[L]=[l R]=0, \quad[R]=R_{i}(X) \quad\left(-X_{\min }<X<X_{\max }\right)
$$

where $R_{i}(X)$ is some initial distribution of signaling receptors.

To reduce the number of parameters in the problem, we introduce a reference unoccupied receptor concentration level $\bar{R}_{0}$ (to be specified later) and the normalized quantities

$$
\begin{gathered}
t=\frac{D}{X_{\max }^{2}} T, \quad x=\frac{X}{X_{\max }}, \quad x_{m}=\frac{X_{\min }}{X_{\max }} \\
\left\{a, b, r, r_{i}\right\}=\frac{1}{\bar{R}_{0}}\left\{[L],[L R],[R], R_{i}\right\}, \quad \gamma=\bar{R}_{0} \Gamma, \\
\left\{v_{L}, v_{R}, v_{\min }, v_{\max }, \rho_{R}\right\}=\frac{1}{D / X_{\max }^{2}}\left\{\frac{V_{L}}{\bar{R}_{0}}, \frac{V_{R}}{\bar{R}_{0}}, \frac{V_{\min }}{\bar{R}_{0}}, \frac{V_{\max }}{\bar{R}_{0}}, \frac{V_{\min }}{V_{\max }}\right\}, \\
\left\{f_{0}, g_{0}, g_{r}, h_{0}\right\}=\frac{1}{D / X_{\max }^{2}}\left\{k_{o f f}, k_{\mathrm{deg}}, r_{\mathrm{deg}}, k_{\text {on }} \bar{R}_{0}\right\} .
\end{gathered}
$$

In terms of these scaled quantities, we write the IBVP in the following normalized form

$$
\frac{\partial a}{\partial t}=\frac{\partial^{2} a}{\partial x^{2}}-h_{0} a r+f_{0} b+v_{L}(x, t) \quad\left(-x_{m}<x<1\right)
$$




$$
\frac{\partial b}{\partial t}=h_{0} a r-\left(f_{0}+g_{0}\right) b, \quad \frac{\partial r}{\partial t}=v_{R}(x, t)-h_{0} a r-g_{r} r+f_{0} b \quad\left(-x_{m} \leq x \leq 1\right)
$$

with

$$
x=-x_{m}: \frac{\partial a}{\partial x}=0, \quad x=1: a=0
$$

for $t>0$ and

$$
t=0: a=b=0, \quad r=r_{i}(x) \quad\left(-x_{m}<x<1\right)
$$

where

$$
\begin{aligned}
v_{R} & =\frac{V_{\max } / \bar{R}_{0}}{D / X_{\max }^{2}}\left[\rho_{R}+\frac{1-\rho_{R}}{1+\gamma b^{n}}\right] \\
& =v_{\max }(x, t)\left[\frac{1+\rho_{R} \gamma b^{n}}{1+\gamma b^{n}}\right] \equiv v_{\max }(x, t) f_{R}(b),
\end{aligned}
$$

and

$$
0 \leq \rho_{R}=\frac{V_{\min }(X, T)}{V_{\max }(X, T)}=\frac{v_{\min }(x, t)}{v_{\max }(x, t)} \leq 1
$$

\section{Time Independent Steady State Behavior}

\subsection{Reduction of the Steady State Equations}

For cells to express differentially, it is important that the signaling morphogen concentrations in the wing imaginal disc evolves toward to a time independent steady state with a reasonable slope and convexity. For the present investigation, it suffices to consider a localized $D p p$ synthesis rate in the form of a step function with $V_{L}(X, T)=\bar{V}_{L} H(-X)$ for some constant $\bar{V}_{L}$. Correspondingly, we have

$$
v_{L}(x, t)=v_{L}(x)=\bar{v}_{L} H(-x)=\left\{\begin{array}{c}
\bar{v}_{L} \\
0
\end{array}, \quad \bar{v}_{L}=\frac{\bar{V}_{L} / \bar{R}_{0}}{D / X_{\max }^{2}} .\right.
$$

We will also consider uniform maximum and minimum receptor synthesis rates with

$$
\left\{V_{\max }(X, T), V_{\min }(X, T)\right\}=\left\{\bar{V}_{\max }, \bar{V}_{\min }\right\}
$$

so that the normalized nonnegative receptor synthesis rate $v_{R}$ takes the form

$$
v_{R}(x, t)=\frac{\bar{V}_{\max } / \bar{R}_{0}}{D / X_{\max }^{2}} f_{R}(b) \equiv \bar{v}_{\max } f_{R}(b)
$$

where we have now a constant ratio of the two limit receptor synthesis rates: $\rho_{R}=$ $\bar{V}_{\min } / \bar{V}_{\max } \equiv \bar{v}_{\min } / \bar{v}_{\max }$. Note that the case of different receptor synthesis rates in and 
outside the morphogen production zone has been examined in previous publications $[7,8]$. Here we focus only on the case where $V_{\min }$ and $V_{\max }$ are constants so that, in the absence of feedback, the receptor synthesis rate is uniform throughout the posterior compartment (given that we have $f_{R}(b)=1$ for $\gamma=0$ ).

With the initial receptor concentration taken to be the steady state receptor distribution prior to the onset of morphogen production, $R_{i}(x)=\left[V_{R}(X) / r_{\mathrm{deg}}\right]_{[L R]=0}$, we set

$$
\bar{R}_{0}=\frac{\bar{V}_{\max }}{r_{\text {deg }}}
$$

so that we have from (16)

$$
\bar{v}_{\max }=g_{r}, \quad R_{i}(x)=\bar{R}_{0} r_{i}(x) .
$$

For our choice of receptor synthesis rate $V_{R}(X)$, we have

$$
r_{i}(x)=\left[\frac{V_{R}(X)}{\bar{V}_{\max }}\right]=1
$$

given that we have taken $V_{R}(X)=V_{\max }(X)=\bar{V}_{\max }$ in the absence of bound morphogen concentration $(b=0)$.

We are interested in a time independent steady state solution

$$
\{a(x, t), b(x, t), r(x, t)\}=\{\bar{a}(x), \bar{b}(x), \bar{r}(x)\}
$$

for the system (9) - (12). For such a solution, we may set all time derivatives in these equations to zero to get

$$
\begin{gathered}
0=\bar{a}^{\prime \prime}-h_{0} \bar{a} \bar{r}+f_{0} \bar{b}+v_{L}(x), \\
0=h_{0} \bar{a} \bar{r}-\left(f_{0}+g_{0}\right) \bar{b}, \quad 0=g_{r} f_{R}(\bar{b})-h_{0} \bar{a} \bar{r}-g_{r} \bar{r}+f_{0} \bar{b},
\end{gathered}
$$

where a prime indicates differentiation with respect to $x$. The nonlinear second order system of ODE (25) - (26) is augmented by the boundary conditions

$$
\bar{a}\left(-x_{m}\right)=0, \quad \bar{a}(1)=0 .
$$

With $v_{L}(x)$ being piecewise constant, the form of $(25)$ - (26) requires that $\bar{a}(x)$ and its first derivative to be continuous at $x=0$.

In previous investigations without feedback $[7,8,9,10]$, the two equations in (26) were solved for $\bar{b}$ and $\bar{r}$ in terms of $\bar{a}$ and the results used to reduce (25) to a single ODE for $\bar{a}$. For the present problem, we can solve the first equation in (26) to get $\bar{r}=\alpha_{0} \bar{b} / \bar{a}$. Upon substituting this in the second equation of (26), we obtain a polynomial equation in $\bar{b}$ with $\bar{a}$ in the various coefficients:

$$
\begin{aligned}
P(\bar{b}) & \equiv \gamma A(\bar{a}) \bar{b}^{n+1}-\gamma B(\bar{a}) \bar{b}^{n}+A(\bar{a}) \bar{b}-[B(\bar{a})+C(\bar{a})] \\
& =(A \bar{b}-B)\left(\gamma \bar{b}^{n}+1\right)-C=0
\end{aligned}
$$


where

$$
\begin{aligned}
& A(\bar{a})=g_{0} h_{0} \bar{a}+g_{r}\left(f_{0}+g_{0}\right) \equiv A_{1} \bar{a}+A_{0}, \\
& B(\bar{a})=h_{0} \bar{v}_{\min } \bar{a} \equiv B_{1} \bar{a}, \quad C(\bar{a})=h_{0} \bar{a}\left(\bar{v}_{\max }-\bar{v}_{\text {min }}\right) \equiv C_{1} \bar{a} .
\end{aligned}
$$

Except for the special case $n=1$, the relation (28) cannot be used to express $\bar{b}$ explicitly in terms of $\bar{a}$.

On the other hand, $P(\bar{b})$ is linear in $\bar{a}$ so that we can use (28) to express $\bar{a}$ in terms of $\bar{b}$ :

$$
\bar{a}=\frac{\alpha_{0} \bar{b}}{f_{R}(\bar{b})-\varsigma \bar{b}}
$$

where

$$
\alpha_{0}=\frac{f_{0}+g_{0}}{h_{0}}, \quad \varsigma=\frac{g_{0}}{g_{r}} .
$$

The expression (31) can then be used to eliminate $\bar{a}$ from (25) to get a single second order ODE for $\bar{b}$ :

$$
\left[\frac{\alpha_{0} \bar{b}}{f_{R}(\bar{b})-\varsigma \bar{b}}\right]^{\prime \prime}-g_{0} \bar{b}+v_{L}(x)=0 \quad\left(-x_{m}<x<1\right),
$$

keeping in mind that $f_{R}(\bar{b})$ depends on $x$ through $\bar{b}(x)$ (see (16)). Unfortunately, the form of this ODE is awkward both for theoretical analysis and numerical solutions even if the boundary conditions for $\bar{b}$ also take relatively simple forms:

$$
\bar{b}\left(-x_{m}\right)=0, \quad \bar{b}(1)=0 .
$$

\subsection{Existence of a Unique Set of Steady State Gradients}

Whether we work with $\bar{a}(x)$ or $\bar{b}(x)$ as the primary unknown, it is not enough to compute solutions of the BVP governing the steady state gradients for some sets of values for the system rate constants. Biologists want to be assured that such steady state gradients exist for any biologically realistic set of parameter values. For this and other reasons, we will show in this section that there is a unique solution of the relevant BVP for the steady state morphogen concentrations. Since the auxiliary conditions are naturally prescribed in terms of the free morphogen concentration, we will stay with the unknown $\bar{a}(x)$ and take $\bar{b}(x)$ and $\bar{r}(x)$ in terms of $\bar{a}(x)$. For this purpose, we need the following preliminary result:

Lemma 1 For a fixed $\gamma$ and $n$, there exists a unique $\bar{b}$ in $(B(\bar{a}) / A(\bar{a}), \infty)$ for any nonnegative $\bar{a}$, denoted by $b^{*}(\bar{a})$, which is an increasing function of $\bar{a}$.

Proof. Since $\bar{b}$ is non-negative, we have from $(28) \bar{b}=b^{*}(0)=0$ and $P(\bar{b}) \leq$ $-C(\bar{a}) \leq 0$ for $\bar{a}>0$ and $0<\bar{b} \leq B(\bar{a}) / A(\bar{a})$. For larger values of $\bar{b}, P(\bar{b})$ is strictly increasing for all $\bar{b}$ in $(B(\bar{a}) / A(\bar{a}), \infty)$ with $P(\bar{b}) \rightarrow \infty$ as $\bar{b} \rightarrow \infty$. Since 
$P(\bar{b})$ is a polynomial, we have by intermediate value theorem a value $b^{*}$ in $(B / A, \infty)$, depending on $\bar{a}$, for which $P(\bar{b})$ vanishes, i.e., $P\left(b^{*}\right)=0$. Furthermore, $\bar{b}=b^{*}(\bar{a})$ is unique for any $\bar{a}(x) \geq 0$ by the monotonicity of $P(\bar{b})$ in $(B / A, \infty)$.

Lemma 2 For fixed $\gamma$ and $n, b^{*}(\bar{a})$ is an increasing function of $\bar{a}$ for all $\bar{a}>0$.

Proof. To show that $\bar{b}=b^{*}(\bar{a})$ is an increasing function of $\bar{a}$, we differentiate (28) with respect to $\bar{a}$ to get

$$
\frac{d b^{*}}{d \bar{a}}=\frac{A_{0}\left(1+\gamma \bar{b}^{n}\right)}{\Delta} \frac{\bar{b}}{\bar{a}}, \quad \Delta=\frac{\left(1+\gamma \bar{b}^{n}\right)}{\left(1+\gamma \bar{b}^{n}\right)^{2} A+n \gamma \bar{b}^{n-1} C} .
$$

Since the right side of the first relation above is positive for $\bar{b}$ in $(B / A, \infty)$, the second part of the lemma is proved.

With Lemma 1 above, we can write

$$
\begin{array}{rlc}
-\bar{a}^{\prime \prime}+g_{0} b^{*}(\bar{a})-v_{L}(x) & =0 & \left(-x_{m}<x<1\right), \\
\bar{a}^{\prime}\left(-x_{m}\right) & =0, & \bar{a}(1)=0
\end{array}
$$

where $b^{*}(\bar{a})$ a well-defined continuous and monotone function of $\bar{a}$. Hence we have a well-defined BVP for $\bar{a}(x)$ for which we will show presently that there is a unique monotone solution.

Proposition 3 The BVP (36)-(37) has a nonnegative solution $\bar{a}(x) \geq 0$.

Proof. The existence of a nonnegative solution of the boundary value problem is proved by producing a nonnegative upper solution and a nonnegative lower solution for the problem. From (28), we have $P(\bar{b}) \equiv(A \bar{b}-B)\left(\gamma \bar{b}^{n}+1\right)=C$ so that $\bar{b} \leq(B+C) / A$ and therewith

$$
-a_{\ell}^{\prime \prime}+g_{0} \frac{B+C}{A}-v_{\ell}(x) \leq-a_{\ell}^{\prime \prime}+g_{0} b^{*}\left(a_{\ell}\right)-v_{\ell}(x) \quad\left(-x_{m}<x<1\right) .
$$

Hence, a lower solution $a_{\ell}$ of the BVP for $\bar{a}$ is therefore given by the solution of

$$
-a_{\ell}^{\prime \prime}+g_{0} \frac{B\left(a_{\ell}\right)+C\left(a_{\ell}\right)}{A\left(a_{\ell}\right)}-v_{\ell}(x)=0, \quad a_{\ell}^{\prime}\left(-x_{m}\right)=0, \quad a_{\ell}(1)=0 .
$$

For an upper solution, we note from $C \geq 0$ and (28) that $b(a) \geq B(a) / A(a)$ so that

$$
-a_{u}^{\prime \prime}+g_{0} \frac{B\left(a_{u}\right)}{A\left(a_{u}\right)}-v_{\ell}(x) \geq-a_{u}^{\prime \prime}+g_{0} b^{*}\left(a_{u}\right)-v_{\ell}(x) \quad\left(-x_{m}<x<1\right) .
$$


An upper solution $a_{u}$ of the BVP for $\bar{a}$ is therefore given by the solution of the

$$
-a_{u}^{\prime \prime}+g_{0} \frac{B\left(a_{u}\right)}{A\left(a_{u}\right)}-v_{\ell}(x)=0, \quad a_{u}^{\prime}\left(-x_{m}\right)=0, \quad a_{u}(1)=0 .
$$

By the results of [9], we know both $a_{\ell}(x)$ and $a_{u}(x)$ exist and are nonnegative. A theorem of D.H. Sattinger established in [14] (see also [1, 15]) assures the existence of a nonnegative $\bar{a}(x)$ for the $\operatorname{BVP}(36)$ - (37) with

$$
0 \leq a_{\ell}(x) \leq \bar{a}(x) \leq a_{u}(x)
$$

Proposition 4 The solution of the BVP for $\bar{a}(x)$ is unique.

Proof. Let $a_{1}(x)$ and $a_{2}(x)$ be two (nonnegative) solutions and $a(x)=a_{1}(x)-$ $a_{2}(x)$. Then as a consequence of the differential equation (33) for $a_{1}(x)$ and $a_{2}(x)$, the difference $a(x)$ satisfies the following differential equation:

$$
-a^{\prime \prime}+g_{0}\left[b^{*}\left(a_{1}\right)-b^{*}\left(a_{2}\right)\right]=-a^{\prime \prime}+g_{0} b^{\cdot}(\xi) a=0
$$

where $b^{*}(a)=d b^{*} / d a$ and where we have used the mean value theorem for some intermediate value $\xi$ between $a_{1}$ and $a_{2}$. Form

$$
\int_{-x_{m}}^{1}\left[-a^{\prime \prime}+g_{0} b(\xi) a\right] a(x) d x=0 .
$$

Upon integration by parts and applications of the boundary conditions in (37), the relation above may be transformed into

$$
\int_{-x_{m}}^{1}\left[a^{\prime}\right]^{2} d x+g_{0} b(\xi) \int_{-x_{m}}^{1}[a(x)]^{2} d x=0 .
$$

Given Lemma 2, the left side of the equation above is nonnegative and vanishes only if $a(x) \equiv 0$ and hence uniqueness.

\subsection{Monotonicity}

Proposition $5 \bar{a}(x)$ is monotone decreasing in $(0,1)$.

Proof. First, we prove that $\bar{a}(x)$ does not have a local maximum in $(0,1)$. If an interior maximum occurs at $x_{0}$, then $a^{\prime}\left(x_{0}\right)=0$ and $a^{\prime \prime}\left(x_{0}\right) \leq 0$. But we have from (36) $\bar{a}^{\prime \prime}\left(x_{0}\right)=g_{0} b^{*}(\bar{a}) \geq 0$. Hence, we must have $a^{\prime \prime}\left(x_{0}\right)=0$ and therewith $b^{*}\left(\bar{a}\left(x_{0}\right)\right)=0$. In that case (28) reduces to $B\left(x_{0}\right)+C\left(x_{0}\right)=0$ or, with the expressions for $\mathrm{B}$ and $\mathrm{C}$ in $(30), \bar{a}\left(x_{0}\right)=0$. But $\bar{a}\left(x_{0}\right)=0$ is a local maximum and $\bar{a}(x) \geq 0$, it follows 
that $\bar{a}(x)=0$ for all $x$ in $(0,1)$. But continuity conditions on $\bar{a}$ and $\bar{a}^{\prime}$ at $x=0$ determines $\bar{a}(x)$ for $\left(-x_{m}, 0\right)$, contradicting the ODE for $\bar{a}(x)$ in that interval where $v_{L}(x)$ does not vanish identically.

On the other hand, $\bar{a}(x)$ does not have a local minimum in $(0,1)$. If it should attain a minimum at $x_{1}$, then $\bar{a}(x)$ must attain a maximum at a point $x_{2}$ in $\left(x_{1}, 1\right)$ or $\bar{a}(x)=0$ in $(0,1)$, given $\bar{a}(x) \geq 0$ and $\bar{a}(1)=0$. Neither is possible. Thus, $\bar{a}(x)$ must be monotone in $(0,1)$. Since $\bar{a}(1)=0, \bar{a}(x)$ must be monotone decreasing.

Proposition $6 \bar{a}(x)$ is monotone in $\left(-x_{m}, 0\right)$.

Proof. First, $\bar{a}(x)$ does not have a local maximum in $\left(-x_{m}, 0\right)$. If there should be at least one interior extremum, consider the one closest to $-x_{m}$, say at $-x_{0}$ with $\bar{a}\left(-x_{0}\right)=0$. Then the ODE together with the two auxiliary conditions $\bar{a}\left(-x_{0}\right)=0$ and $\bar{a}(1)=0$, completely determines $\bar{a}(x)$ (uniquely by the result of the previous subsection) in $\left(-x_{0}, 1\right)$. By continuity, the terminal values $\bar{a}\left(-x_{0}\right)$ and $\bar{a}^{\prime}\left(-x_{0}\right)$ determines $\bar{a}(x)$ uniquely in the interval at $\left[-x_{m},-x_{0}\right]$.

If $\bar{a}\left(-x_{0}\right)$ should be a local maximum then the ODE in this interval requires $\bar{a}\left(-x_{m}\right)$ to be a local maximum also. In that case, there must be a local minimum inside the interval $\left(-x_{m},-x_{0}\right)$ which contradicts the stipulation that fact that $\bar{a}\left(-x_{0}\right)$ is closest extremum to the end point $x=-x_{m}$.

If $\bar{a}\left(-x_{0}\right)$ should be a local minimum, then either $\bar{a}(x)$ attains a local maximum at some $x_{1}$ in $\left(-x_{0}, 1\right)$ or $\bar{a}(x) \equiv 0$ there. Neither is possible. The former is impossible given

$$
\bar{a}^{\prime \prime}\left(-x_{1}\right)=g_{0} b^{*}\left(\bar{a}\left(-x_{1}\right)\right)-\bar{v}_{L} \geq g_{0} b^{*}\left(\bar{a}\left(-x_{0}\right)\right)-\bar{v}_{L}=\bar{a}^{\prime \prime}\left(-x_{0}\right) \geq 0
$$

with $\bar{a}\left(x_{1}\right) \geq \bar{a}\left(x_{0}\right)$. The latter is impossible because $v_{L}(x)>0$ in $\left(-x_{m}, 0\right)$. Thus, $\bar{a}(x)$ must be monotone in $\left(-x_{m}, 0\right)$.

Proposition $7 \bar{a}(x)$ is monotone decreasing in $\left(-x_{m}, 1\right)$.

Proof. Since $\bar{a}^{\prime}(0)<0$ by Proposition 5 and $\bar{a}^{\prime}(x)$ is continuous at $x=0$, we must have $\bar{a}^{\prime}(x)<0$ in $\left(-x_{m}, 0\right]$ given that $\bar{a}(x)$ has no interior minimum or maximum there. It follows that $\bar{a}(x)$ is monotone decreasing in $\left(-x_{m}, 0\right)$ and, by Proposition 5 , in the larger interval $\left(-x_{m}, 1\right)$.

\section{Linear Stability}

\subsection{A Nonlinear Eigenvalue Problem}

In addition to the existence of unique steady state concentrations $\bar{a}(x), \bar{b}(x)$, and $\bar{r}(x)$, it is important for these concentrations to be asymptotically stable. To investigate 
the stability of the steady state solution known to exist from Theorem 3, we consider small perturbations from the steady state solution in the form

$$
\{a(x, t), b(x, t), r(x, t)\}=\{\bar{a}(x), \bar{b}(x), \bar{r}(x)\}+e^{-\lambda t}\{\hat{a}(x), \hat{b}(x), \hat{r}(x)\} .
$$

After linearization, the differential equations (12)-(13) become

$$
\begin{aligned}
-\lambda \hat{a} & =\hat{a}^{\prime \prime}-h_{0}(\bar{r} \hat{a}+\bar{a} \hat{r})+f_{0} \hat{b}, \\
-\lambda \hat{b} & =h_{0}(\bar{r} \hat{a}+\bar{a} \hat{r})-\left(f_{0}+g_{0}\right) \hat{b}, \\
-\lambda \hat{r} & =-h_{0}(\bar{r} \hat{a}+\bar{a} \hat{r})-g_{r} \hat{r}+\left[f_{0}-p(\bar{b})\right] \hat{b},
\end{aligned}
$$

where

$$
p(b)=n \gamma\left(\bar{v}_{\max }-\bar{v}_{\min }\right) \frac{\bar{b}^{n-1}}{\left(1+\gamma \bar{b}^{n}\right)^{2}} \geq 0 .
$$

The relations (40) and (41) are then solved for $\hat{b}$ and $\hat{r}$ in terms of $\hat{a}$ to get

$$
\begin{aligned}
\hat{r} & =\frac{h_{0} \bar{r}(x)\left[\lambda-p(\bar{b})-g_{0}\right] \hat{a}}{\left(g_{r}-\lambda\right)\left(f_{0}+g_{0}-\lambda\right)+h_{0} \bar{a}(x)\left[g_{0}-\lambda+p(\bar{b})\right]}, \\
\hat{b} & =\frac{h_{0} \bar{r}(x)\left(g_{r}-\lambda\right) \hat{a}}{\left(g_{r}-\lambda\right)\left(f_{0}+g_{0}-\lambda\right)+h_{0} \bar{a}(x)\left[g_{0}-\lambda+p(\bar{b})\right]} .
\end{aligned}
$$

The expressions (43) and (44) are used to eliminate $\hat{b}$ and $\hat{r}$ from (39) to obtain

$$
\hat{a}^{\prime \prime}+\left[\lambda-q_{r}(x ; \lambda)\right] \hat{a}=0,
$$

where

$$
q_{r}(x ; \lambda)=\frac{h_{0} \bar{r}(x)\left(g_{r}-\lambda\right)\left(g_{0}-\lambda\right)}{\left(g_{r}-\lambda\right)\left(g_{0}+f_{0}-\lambda\right)+h_{0} \bar{a}(x)\left[g_{0}-\lambda+p(\bar{b})\right]} .
$$

The ODE for $\hat{a}(x)$ is supplemented by the boundary conditions

$$
\hat{a}^{\prime}\left(-x_{m}\right)=0, \quad \hat{a}(1)=0 .
$$

Together, (45) and (46) define an eigenvalue problem with $\lambda$ as the eigenvalue parameter. Though the ODE is linear, the eigenvalue problem is nonlinear since $\lambda$ appears nonlinearly in $q_{r}(x ; \lambda)$ so that $(45)$ and (46) is not a Sturm-Liouville problem. In the next subsection, we will show that the eigenvalues of the homogeneous boundary value problem defined by the differential equation (45) and the homogeneous boundary conditions (46) must be positive. It follows then that the steady state gradients are asymptotically stable according to linear stability theory. 


\subsection{Positive Eigenvalues and Asymptotic Stability}

We will prove linear stability of the steady state solution in two steps. First, we prove that the eigenvalues of (45) and (46) are real. These real eigenvalues are then proved to be positive.

Lemma 8 All the eigenvalues of the nonlinear eigenvalue problem (45) and (46) are real.

Proof. Suppose $\lambda$ is a complex eigenvalue and $a_{\lambda}(x)$ an associated nontrivial eigenfunction, then $\lambda^{*}$ is also an eigenvalue with eigenfunction $a_{\lambda}^{*}(x)$ where ()$^{*}$ is the complex conjugate of ( ). The bilinear relation

$$
\int_{-x_{m}}^{1}\left[\left(a_{\lambda}^{*}\right) a_{\lambda}^{\prime \prime}-\left(a_{\lambda}^{*}\right)^{\prime \prime} a_{\lambda}\right] d x=0
$$

(which can be established by integration by parts and applications of the boundary conditions in (46)) requires

$$
\int_{-x_{m}}^{1}\left\{\left(\lambda-\lambda^{*}\right)-\left[q_{r}(x ; \lambda)-q_{r}\left(x ; \lambda^{*}\right)\right]\right\}\left(a_{\lambda}^{*} a_{\lambda}\right) d x=0 .
$$

It is straightforward to verify that $q_{r}(x ; \lambda)-q_{r}\left(x ; \lambda^{*}\right)=-\left(\lambda-\lambda^{*}\right) \Phi\left(x ; \lambda \lambda^{*}\right)$, where

$$
\Phi(x ; \lambda)=\frac{f_{0}\left|g_{r}-\lambda\right|^{2}+h_{0} \bar{a}(x)\left|g_{0}-\lambda\right|^{2}+h_{0} \bar{a}(x) p(\bar{b})\left[g_{0}+g_{r}+2 \operatorname{Re}(\lambda)\right]}{\left|\left(g_{r}-\lambda\right)\left(g_{0}+f_{0}-\lambda\right)+h_{0} \bar{a}(x)\left[g_{0}-\lambda+p(\bar{b})\right]\right|^{2}},
$$

is a positive real value function of $\lambda$, given the definition of $p(x)$ in (42). In that case, the condition (47) becomes

$$
\left(\lambda-\lambda^{*}\right) \int_{-x_{m}}^{1} a_{\lambda} a_{\lambda}^{*}[1+\Phi(x ; \lambda)] d x=0 .
$$

Since the integral is positive for any nontrivial $a_{\lambda}(x)$, we must have $\lambda-\lambda^{*}=0$. Hence, $\lambda$ does not have an imaginary part.

Theorem 9 All eigenvalues of the nonlinear eigenvalue problem (39)-(41) and (46) are positive and the steady state concentrations $\bar{a}(x), \bar{b}(x)$ and $\bar{r}(x)$ are asymptotically stable with respect to small perturbations from the steady state.

Proof. Suppose $\lambda \leq 0$. Let $\hat{a}_{\lambda}(x)$ be a nontrivial eigenfunction of the homogeneous BVP (45) and (46) for the nonpositive eigenvalue $\lambda$. Multiply (45) by $\hat{a}_{\lambda}$ and integrate over the solution domain to get

$$
\int_{-x_{m}}^{1}\left\{\hat{a}_{\lambda} \hat{a}_{\lambda}^{\prime \prime}-q_{r}(x ; \lambda)\left(\hat{a}_{\lambda}\right)^{2}\right\} d x=-\lambda \int_{-x_{m}}^{1}\left(\hat{a}_{\lambda}\right)^{2} d x .
$$


After integration by parts and applications of the homogeneous boundary conditions (46), we obtain

$$
\lambda \int_{-x_{m}}^{1}\left(\hat{a}_{\lambda}\right)^{2} d x=\int_{-x_{m}}^{1}\left(\hat{a}_{\lambda}^{\prime}\right)^{2} d x+\int_{-x_{m}}^{1} q_{r}(x ; \lambda)\left(\hat{a}_{\lambda}\right)^{2} d x .
$$

With $\lambda=-|\lambda| \leq 0$, we have

$$
q_{r}(x ;-|\lambda|)=\frac{\bar{r}(x) h_{0}\left(g_{0}+|\lambda|\right)\left(g_{r}+|\lambda|\right)}{\left(g_{r}+|\lambda|\right)\left(g_{0}+f_{0}+|\lambda|\right)+h_{0} \bar{a}(x)\left[g_{0}+|\lambda|+p(\bar{b})\right]} \geq 0
$$

given the definition of $p(x)$ in (42). For any nontrivial solution of the eigenvalue problem under the assumption $\lambda \leq 0$, the right-hand side of (50) is positive which contradicts the assumption $\lambda=-|\lambda| \leq 0$. Hence the eigenvalues of the eigenvalue problem (45) and (46) must be positive and the theorem is proved.

\subsection{A Rayleigh Quotient}

Similar to the case of no feedback in [9], we want to know the actual magnitude of the smallest eigenvalue to give some idea of how quickly the system returns to steady state after small perturbations. As parametric studies require that we repeatedly compute the time evolution of the concentration of both free and bound morphogens from their initial conditions, the value of the smallest eigenvalue will also give some idea of the decay rate of the transient behavior and thereby the time to reach steady state.

Let $\lambda(>0)$ be an eigenvalue of (45) and (46) and $\hat{a}_{\lambda}(x)$ the corresponding eigenfunction. Upon multiplying ODE (45) for this eigen-pair by $\hat{a}_{\lambda}(x)$ and integrating by parts, we obtain the following Rayleigh quotient-like relation for $\lambda$ after observing the boundary conditions (46) which apply to $\hat{a}_{\lambda}(x)$ :

$$
\lambda \int_{-x_{m}}^{1}\left(\hat{a}_{\lambda}\right)^{2} d x=\int_{-x_{m}}^{1}\left(\hat{a}_{\lambda}^{\prime}\right)^{2} d x+\int_{-x_{m}}^{1} q_{r}(x ; \lambda)\left(\hat{a}_{\lambda}\right)^{2} d x .
$$

The following key result can be proved similar to that in [9]:

Lemma 10 There exists some $\xi=\xi(\lambda)$ in $(0,1)$ for which

$$
\lambda \int_{-x_{m}}^{1}\left(\hat{a}_{\lambda}\right)^{2} d x=\int_{-x_{m}}^{1}\left(\hat{a}_{\lambda}^{\prime}\right)^{2} d x+q_{r}(\xi ; \lambda) \int_{-x_{m}}^{1}\left(\hat{a}_{\lambda}\right)^{2} d x
$$

or, in the form of a Rayleigh quotient,

$$
\Lambda \int_{-x_{m}}^{1}\left(\hat{a}_{\lambda}\right)^{2} d x=\int_{-x_{m}}^{1}\left(\hat{a}_{\lambda}^{\prime}\right)^{2} d x, \quad \text { with } \Lambda=\lambda-q_{r}(\xi, \lambda) .
$$


With the auxiliary conditions (46), it is well known that the minimum value of $\Lambda$ is

$$
\Lambda_{s}=\left(\frac{\pi / 2}{1+x_{m}}\right)^{2}
$$

attained when $\hat{a}_{\lambda}(x)$ is a multiple of the corresponding eigenfunction $\sin \left(\sqrt{\Lambda_{s}}(1-x)\right)$ [19]. It follows that we have

$$
\Lambda \geq \Lambda_{s}
$$

since the actual solution $\hat{a}_{\lambda}(x)$ of (45) and (46) is not $\sin \left(\sqrt{\Lambda_{s}}(1-x)\right)$. In fact, if we let $\lambda_{s}$ denote the smallest eigenvalue of the eigenvalue problem (45) and (46). Then we have also

\section{Lemma $11 \Lambda\left(\lambda_{s} ; \xi\right) \geq \Lambda_{s}$.}

What we really want to know is the smallest eigenvalue $\lambda_{s}$ of the nonlinear eigenvalue problem (45) and (46) which determines the decay rate of transients. Unfortunately, strict inequality generally holds in Lemma 11. Even if we have equality instead of inequality, it is still not possible to solve for $\lambda_{s}$ because we do not know $\xi$ (which depends on $\lambda_{s}$ ). Our goal will have to be a more modest one of finding some useful upper and lower bound(s) for the smallest eigenvalue $\lambda_{s}$. The obvious lower bound, $\lambda_{s}>0$ (which we know from the previous subsection), is not particularly helpful. More useful bounds have been obtained in [6] by methods similar to those used in [9].

\section{$5 \quad$ Some Steady State Gradients}

\subsection{Simplification for $n=1$}

Whether we take the primary dependent variable as $\bar{a}$ or $\bar{b}$, it is not possible to obtain a useful exact solution of the BVP for the steady state solution in terms of known functions even when the morphogen synthesis rate is piecewise uniform. In this section, we obtain one relatively tractable solution for $n=1$ to provide some insight to the effect of negative feedback on the steady state morphogen gradients. The qualitative conclusions can be extended to the general case with the help of the theoretical results of Subsection 3.3.

In varying degree of severity, the main obstacle in obtaining any kind of solution when the problem is formulated in terms of $\bar{a}$ is our inability to express $\bar{b}$ in terms of $\bar{a}$. This obstacle does not exist for the case $n=1$. In that case, we can solve (28), $P(\bar{b})=0$, which is now a quadratic equation for $\bar{b}$, to get the following expression for $\bar{b}$ in terms of $\bar{a}$ :

$$
\bar{b}(x)=b^{*}(\bar{a})=\frac{-\left|\alpha_{0}+\varsigma_{\gamma} \bar{a}\right|+\sqrt{\left(\alpha_{0}+\varsigma_{\gamma} \bar{a}\right)^{2}+4 \gamma \bar{a}\left(\alpha_{0}+\varsigma \bar{a}\right)}}{2 \gamma\left(\alpha_{0}+\varsigma \bar{a}\right)},
$$


with

$$
\varsigma_{\gamma}=\varsigma-\gamma \rho_{R}=\frac{g_{0}}{g_{r}}-\gamma \frac{\bar{V}_{\min }}{\bar{V}_{\max }} .
$$

(We have used $\left|\alpha_{0}+\varsigma_{\gamma} \bar{a}\right|$ to anticipate the possibility of $\alpha_{0}+\varsigma_{\gamma} \bar{a}$ may be negative for sufficiently large $\gamma$.) With (55) and the first relation in (26), equation (25) may then be written as a single ODE for $\bar{a}$ with the help of $\bar{b}(x)$ :

$$
\bar{a}^{\prime \prime}-g_{0} b^{*}(\bar{a})+v_{L}(x)=0 \quad\left(-x_{m}<x<1\right) .
$$

The second order ODE (57) is supplemented by the two boundary conditions (37), keeping in mind also the continuity conditions on $\bar{a}$ and $\bar{a}^{\prime}$ at $x=0$.

Note that for sufficiently small $\gamma$ so that $4 \gamma \bar{a}\left(\alpha_{0}+\varsigma \bar{a}\right) /\left(\alpha_{0}+\varsigma_{\gamma} \bar{a}\right)^{2} \ll 1$, we have as a two-term Taylor approximation

$$
\bar{b}(x)=b^{*}(\bar{a}) \sim \frac{\bar{a}}{\alpha_{0}+\varsigma_{\gamma} \bar{a}}\left\{1-\frac{2 \gamma \bar{a}\left(\alpha_{0}+\varsigma \bar{a}\right)}{\left(\alpha_{0}+\varsigma_{\gamma} \bar{a}\right)^{2}}\right\} .
$$

The first term corresponds to the case of no feedback and the ODE (57) reduces to the corresponding governing ODE in [9] for $\gamma=0$. The effects of a small $\gamma$ is characterized by the second term shown the consequence of a small feedback on the receptor synthesis rate.

\subsection{Exact Solution}

For our choice of synthesis rates $V_{L}$ and $V_{R}$, we have $v_{L}=0$ in the range $0<x<1$ so that

$$
\bar{a}^{\prime \prime}=g_{0} b^{*}(\bar{a}) \quad(0<x<1) .
$$

The ODE (59) admits a first integral. The auxiliary condition $\bar{a}(1)=0$ determine the constant of integration to give

$$
\left[\bar{a}^{\prime}(x)\right]^{2}-s_{1}^{2}=g_{0} I(\bar{a}), \quad(x>0)
$$

with

$$
\frac{d I(\bar{a})}{d \bar{a}}=\bar{b}(\bar{a}), \quad[I(\bar{a})]_{x=1}=I(0)=0, \quad s_{1}=\bar{a}^{\prime}(1) .
$$

Similarly, we have for the interval $\left(-x_{m}, 0\right)$

$$
\left[\bar{a}^{\prime}(x)\right]^{2}=g_{0}\left[I(\bar{a})-I\left(a_{m}\right)\right] \quad(x<0), \quad a_{m}=\bar{a}\left(-x_{m}\right) .
$$

In (60) and (62), $s_{1}$ and $a_{m}$ are two unknown constants to be determined by the solution process. The continuity of $\bar{a}^{\prime}(x)$ and $\bar{a}(x)$ at $x=0$ requires

$$
g_{0} I\left(a_{m}\right)+s_{1}^{2}=0 .
$$


The first order ODE (60) and (62) can be integrated once more to give

$$
\int_{\bar{a}}^{a_{0}} \frac{d a}{\sqrt{I(a)+s_{1}^{2} / g_{0}}}=\sqrt{g_{0}} x \quad(0<x<1), \quad a_{0}=\bar{a}(0)
$$

and

$$
\int_{\bar{a}}^{a_{m}} \frac{d a}{\sqrt{I(a)-I\left(a_{m}\right)}}=\sqrt{g_{0}}\left(x+x_{m}\right) \quad\left(-x_{m}<x<0\right) .
$$

It remains to determine $s_{1}$ and $a_{0}$ by the continuity condition on $\bar{a}$ at $x=0$ :

$$
\int_{a_{0}}^{a_{m}} \frac{d a}{\sqrt{I(a)-I\left(a_{m}\right)}}=\sqrt{g_{0}}\left(x_{m}\right)
$$

and the end condition $\bar{a}(1)=0$ :

$$
\int_{0}^{a_{0}} \frac{d a}{\sqrt{I(a)+s_{1}^{2} / g_{0}}}=\sqrt{g_{0}}
$$

The relations (63), (67) and (66) are three conditions for determining the three unknown constants $a_{0}, a_{m}$ and $s_{1}$ to complete the solution process.

\subsection{A Narrow Ligand Source Width}

A significant simplification of the solution can be attained when $X_{\min }$ is small compared to $X_{\max }$. In that case, we may, to a good first approximation, take $X_{\max }$ to be infinite. (Correspondingly, we should use some other reference length $X_{0}$, such as $X_{\min }$ or the typical span of the posterior compartment which is about 100 cell deep, instead of $X_{\max }$ in (8) - (11).) For this approximation, the end condition at $x=1$ is replaced by

$$
\lim _{x \rightarrow \infty} \bar{a}(x)=0, \quad \lim _{x \rightarrow \infty} \bar{a}^{\prime}(x)=0 .
$$

The second condition requires $s_{1}^{2}=0$ so that the condition (63) simplifies to an equation that determines a single unknown $a_{m}$ :

$$
I\left(a_{m}\right)=0
$$

The condition (66) then becomes an equation for determining $a_{0}$ alone.

Even with these simplifications, the values for the two unknown parameters $a_{m}$ and $a_{0}$ must still be obtained numerically by some iterative methods. For each iteration, the values of the two integrals involved must be calculated numerically by an appropriate quadrature formula. Though it is theoretically gratifying to have an exact solution for the problem, the actual solution process is no simpler than to solve the original BVP in the interval $\left(-x_{m}, 1\right)$ directly by some suitable numerical method. For our relatively simple BVP for a single second order ODE of the form $y^{\prime \prime}=f(y)$, 
there exist a number numerical software for its solution in Maple (desolve) or MatLab $(b v p 4 c)$. The same results can also been obtained by numerical integration of the original IBVP (12)-(16) in Section 2. Some accurate numerical simulations of our problem will be reported in the next section for the special case of $n=1$ to provide some insight on the typical effects of our form of negative feedback on the morphogen gradients. They will also serve as background information and motivation for some rigorous results to be obtained there for the general case of $n \geq 1$.

\section{Effects of the Negative Feedback}

To see the effects of the negative feedback of the form (1), we have implemented numerical simulations using Maple desolve (and independently confirmed with Matlab software) for a unit Hill's coefficient, i.e., $n=1$ in (1), and for a set of typical parameter values in Table I below which was used in [9]. Simulations were implemented also for some positive values of the feedback parameter $\gamma$ which did not appear in $[9]$.

\section{Table I: $\quad$ System Parameter Values}

$\begin{array}{ccccccc}X_{\max } & X_{\min } & r_{\text {deg }} & k_{\text {deg }} & k_{\text {off }} & k_{\text {on }} \bar{R}_{0} & \gamma \\ 0.01 \mathrm{~cm} & 10^{-3} \mathrm{~cm} & 10^{-3} / \mathrm{sec} . & 2 \times 10^{-4} / \mathrm{sec} . & 10^{-6} / \mathrm{sec} . & 0.01 / \mathrm{sec} . & 1.0 \\ & & & & & & 2.0 \\ D & V_{L} / \bar{R}_{0} & g_{r} & g_{0} & f_{0} & h_{0} & 10 . \\ 10^{-7} \mathrm{~cm}^{2} / \mathrm{sec} . & 10^{-3} / \mathrm{sec} .^{*} & 1.0 & 0.2 & 0.001 & 10 & 50 . \\ & & & & & & \infty \\ \bar{V}_{\max } & \bar{V}_{\min } & \rho & \rho_{R} & \Delta_{R} & n & 0^{*} \\ 10^{-3} / \mathrm{sec} . & 10^{-4} / \mathrm{sec} . & 1.0 & 0.1 & 0.9 & 1 & \end{array}$

*Normal Dpp synthesis rate (of $V_{L} / \bar{R}_{0}=10^{-3} /$ sec.) to be doubled for cases with feedback.

Figure (1) shows a typical comparison of the computed distributions of $b=$ $[L R] / \bar{R}_{0}$ (for $n=1$ ). The lower dashed curve is the distribution for a normal ligand synthesis rate of $V_{L} / \bar{R}_{0}=10^{-3} / \mathrm{sec}$. without feedback $(\gamma=0)$. The upper dashed curve is a similar distribution with the ligand synthesis rate doubled (again without feedback). Naturally, the concentration of the normalized bound morphogen is much higher for the latter case since $D p p$ is produced at a higher rate. When negative feedback of the form (1) is introduced with $\gamma=1$ for the higher synthesis rate case, the distribution of bound morphogens is changed to the solid curve. While $[L R] / \bar{R}_{0}$ is reduced near the ligand source to a level close to that before doubling the ligand synthesis rate, the shape of the gradient curve is less convex, in fact slightly concave in the important range of $(0,1)$ where the original gradient is appropriately convex.

Figure (2) shows computed distributions of $[L R] / \bar{R}_{0}$ across the posterior compartment for selected values of $\gamma$ (and $n=1$ ). Of the two solid curves, the one that is markedly convex in the interval $(0,1)$ is one for the normal ligand synthesis rate 
without feedback. The corresponding signaling ligand concentrations after doubling ligand synthesis rate and adding feedback with $\gamma=1,2,10$ and 50 are shown as the four (dash and dot) curves in the figure. The magnitude of the signaling ligand concentration near the source region is lower for higher $\gamma$. However, the situation is more complex near the sink edge. While the magnitude of $[L R] / \bar{R}_{0}$ increases slightly as $\gamma$ increases from 1 to 10 , the increase over the previous level occurs in a smaller and smaller interval adjacent to the edge $x=1$. For still larger $\gamma$ (such as $\gamma=50$ ), $[L R] / \bar{R}_{0}$ actually decreases with $\gamma$ for the entire posterior compartment, approaching in the limit as $\gamma$ tends to $\infty$ the solution for $\bar{b}=B / A$ (obtained by taking $\gamma=\infty$ in (28)) given by the lower solid (concave) line curve in the figure.

For the $n=1$ case, the shape of the signaling gradient for any positive $\gamma$ is generally less convex and more concave than the corresponding gradient without feedback $(\gamma=0)$. Though the reduction in convexity is not monotone in $\gamma$ near the sink edge, the general increase in concavity over the $\gamma=0$ case indicates that our negative feedback on receptor synthesis rate leads to a leveling effect on the signaling morphogen gradient. The change in gradient convexity results in undesirable deviations from the target gradient for the original biological pattern and does not promote robustness in development.

The qualitative effects of a low level negative feedback of the form (1) for $n=1$ can also be seen from (58) for a sufficiently small $\gamma$ (not shown in the two figures). Had the free Dpp gradient $\bar{a}(x)$ remained unchanged, the (sufficiently small) negative feedback would reduce the magnitude of the bound morphogen gradient $\bar{b}(x)$ for all $x$ in $\left(-x_{m}, 1\right)$. While this generally contributes to the robustness of the development of the wing imaginal disc, this positive effect is mediated by the differential reduction induced by the spatial variation of the free $D p p$ concentration. With $\bar{a}(x)$ monotone decreasing from $-x_{m}$ to 1 , the percentage reduction of $\bar{b}(x)$ is most severe near the morphogen source and much less substantial adjacent to the wing disc edge. While $\bar{b}(x)$ would remain convex for sufficiently small $\gamma$, this differential magnitude adjustment has the net effect of leveling the gradient in addition to magnitude reduction.

However, the presence of the negative feedback does in fact alter the steady state free $D p p$ concentration gradient and thereby complicates its actual effect on the final steady state signaling (bound) $D p p$ gradient responsible for differential cell expression. To the extent that the negative feedback does not alter the monotone decreasing property of $\bar{a}(x)$, the leveling of the bound $D p p$ gradient by a negative feedback of the form (1) continues to operate, differing only in the degree of severity. Hence, for sufficiently small values of $\gamma$, the particular form of negative feedback (1) tends to reduce cell expression differentially, higher reduction (for the lower receptor synthesis rate) near the source and lower (for the higher receptor synthesis rate) near the edge. Similar to the moderate and high $\gamma$ values, this leveling effect of a low level negative feedback also changes the biologically desirable convexity of bound morphogen gradient and thereby works against robustness of wing disc development. 
These observations (supported by results of numerical solutions of the relevant BVP and by the approximate formula (58)) provide an explanation for the negative simulation results (that feedback of the form (1) does not improve robustness of the biological development of the Drosophila wing imaginal disc). Clearly, a reduction in receptor synthesis rate leads to more $D p p$ available for downstream binding with signaling receptors. However, the differential reduction of $D p p$ synthesis rate induced by a spatially nonuniform bound morphogen concentration changes $\bar{b}(x)$ differentially in space, higher reduction near the source end and lower reduction near the sink edge,resulting in a less convex and (for a sufficiently large $\gamma$ ) possibly concave $\bar{b}(x)$. We will now validate rigorously the aforementioned explanation for the negative simulation results first reported in [12] for the $n=1$ case for the entire range of $\gamma$ values and extend it to the more general case of $n>1$.

By Proposition 7, the steady state free $D p p$ concentration gradient for a fixed $\gamma$ is always monotone decreasing as $x$ increases. By Lemma 2, the change in steady state bound morphogen gradient $\bar{b}(x)$ for a fixed $\gamma$ is also monotone decreasing for increasing $x$ as a consequence. With a spatially nonuniform $\bar{b}(x)$, the leveling effect of our form of negative feedback therefore persists for the general case. However, there are some qualitative differences in this effect for $\gamma$ not sufficiently small so that (58) is not an accurate characterization of the effects of (1). The following results provide a rigorous and more complete characterization of the effect of our negative feedback for a general Hill's coefficient.

Proposition $12 \bar{a}(x ; \gamma)$ is an increasing function of $\gamma$ for $-x_{m}<x<1$.

Proof. Let $w(x)=\partial \bar{a} / \partial \gamma$. Upon differentiating the ODE (36) and BC (37) for $\bar{a}$ partially with respect to $\gamma$, we obtain with the help of (28)-(30)

$$
\begin{array}{rlc}
-w^{\prime \prime}+g_{0} \sigma_{a} w-g_{0} \sigma_{\gamma}=0 & \left(-x_{m}<x<1\right) \\
w^{\prime}\left(-x_{m}\right)=0, & w(1)=0
\end{array}
$$

with

$$
\begin{aligned}
\sigma_{a} & \equiv \frac{\partial b^{*}(\bar{a}, \gamma)}{\partial \bar{a}}=\frac{A_{0} b^{*}}{\Delta \bar{a}} \geq 0, \\
\sigma_{\gamma} & \equiv-\frac{\partial b^{*}(\bar{a}, \gamma)}{\partial \gamma}=\frac{1}{\Delta} \frac{\left(b^{*}\right)^{n} C}{1+\gamma\left(b^{*}\right)^{n}} \geq 0 \\
\Delta & =A\left[1+\gamma\left(b^{*}\right)^{n}\right]+\frac{n \gamma\left(b^{*}\right)^{n-1} C}{1+\gamma\left(b^{*}\right)^{n}}
\end{aligned}
$$

where $A(\bar{a})$ and $C(\bar{a})$ are as defined in (29) and (30). Evidently, $w_{\ell}(x)=0$ is a lower solution of the BVP for $w$. For an upper solution, let $\sigma_{\max }$ be the maximum of $\sigma_{\gamma}(x)$ and $w_{u}(x)$ be the solution of the BVP

$$
\begin{array}{rlc}
-w_{u}^{\prime \prime}+g_{0} \sigma_{a} w_{u}-g_{0} \sigma_{\max }=0 & \left(-x_{m}<x<1\right) \\
w_{u}^{\prime}\left(-x_{m}\right)=0, & w_{u}(1)=0
\end{array}
$$


Then $w_{u}(x)$ is an upper solution of the BVP for $w(x)$. It follows that there exist a unique $w(x)$ that is non-negative. Furthermore $w(x) \equiv 0$ does not satisfy the ODE (70). Hence, the proposition is proved.

For $\bar{b}(x, \gamma)$, we obtain from $(28)-(30)$

$$
\frac{\partial \bar{b}(x, \gamma)}{\partial \gamma}=\frac{g_{r}\left(f_{0}+g_{0}\right)}{\Delta\left(1+\gamma \bar{b}^{n}\right)} \frac{\bar{b}}{\bar{a}}\left\{\left(1+\gamma \bar{b}^{n}\right)^{2} \frac{d \bar{a}}{d \gamma}-\left(1-\rho_{R}\right) \frac{\bar{a}^{2} \bar{b}^{n-1}}{\alpha_{0}}\right\}
$$

where $\Delta$ is as defined in (74) and where the ratio $\bar{b} / \bar{a}$ has a finite limit as $x \rightarrow 1$ :

$$
\lim _{x \rightarrow 1} \frac{\bar{b}(x, \gamma)}{\bar{a}(x, \gamma)}=\frac{B_{1}+C_{1}}{A_{0}}=\frac{1}{\alpha_{0}} .
$$

Unlike the special case of small $\gamma$ and $n=1$, the effects of the feedback parameter $\gamma$ in (1) on the signaling gradient now depends on the sign of the right hand side of (77).

In the source region and nearby, numerical results for the case of $\gamma=1,2,10$ and 50 all lead to a negative value for $\partial \bar{b}(x, \gamma) / \partial \gamma$. Adjacent to the edge sink at $x=1$, the addition of a negative feedback on receptor synthesis rate of the form (1) gives a positive value for right hand side of (77) for $\gamma=1,2$ and 10 with the interval for the positive value shrinking with increasing $\gamma$. For $\gamma>50$, the region of positive right hand side of (77) is imperceptible, consistent with the numerical simulation results showing that beyond a certain critical value of $\gamma$, the signaling morphogen concentration decreases monotonically and pointwise toward the limiting distribution corresponding to the case of $\bar{b}=B(\bar{a}) / A(\bar{a})=B_{1} \bar{a} /\left[A_{1} \bar{a}+A_{0}\right]$. The gradient of this limiting solution is still concave for the $n=1$ case though somewhat less so compared to those for (finite but) large values of $\gamma$. As such the addition of a negative feedback of the form (1) drives the signaling gradient away from its target shape for biological development.

This is not to say that negative feedback mechanisms are generally not effective for robustness. But if robustness is to be achieved, the feedback process should not promote a leveling effect on the convex signaling gradient. Rather, our observations suggest that an effective negative feedback mechanism for robustness through regulating the receptor synthesis rate should be spatially uniform. For example, the desired spatial uniform feedback effect may be attained with a negative feedback on the receptor synthesis rate of the form

$$
V_{R}=V_{\min }+\frac{V_{\max }-V_{\min }}{1+\Gamma([L R])}=V_{\max }\left\{\rho_{R}+\frac{\Delta_{R}}{1+\gamma(\bar{b}(x))}\right\}
$$

where $\gamma(\bar{b}(x))$ is a functional of the signaling gradient $\bar{b}(x)$. Possible choices of $\gamma(\bar{b}(x))$ include $\gamma_{0} \bar{b}(0)$ and some average value of the signaling morphogen concentration,

$$
\gamma(\bar{b}(x))=\gamma_{0} \int_{0}^{1} \bar{b}(x) d x .
$$

The effects of such spatially uniform feedback mechanisms are being investigated. 


\section{Conclusion}

The present research was motivated by the results of [12] on negative feedback as an instrument for achieving robustness in biological developments. The basic models for morphogen gradient formation that accounts only for diffusion, reversible binding with renewable signaling receptors, and degradation (with or without transcytosis of bound morphogens) were found to be sensitive to changes in system parameters. Doubling the morphogen synthesis rate changes the corresponding signaling bound $D p p$ gradient substantially, exceeding the acceptable range of a robustness measure introduced in [12] for all sets of parameter values simulated. Conventional wisdom would have the sensitivity to system characteristics lowered by some form of negative feedback which is expected to ameliorate the response to the system parameter changes. It was therefore rather surprising that a negative feedback mechanism of the form (1) known to be effective in other applications was found not to render the development of the Drosophila wing imaginal disc more robust [12]. In fact, numerical simulations for $10^{6}$ sets of system parameter values showed generally a higher sensitivity to a doubling of $D p p$ synthesis rate than the basic models. The unexpected finding prompted the present examination of the cause of the ineffectiveness of the negative feedback mechanism (1).

From the approximate analysis and numerical simulations for the special case of $n=1$, we found that in addition to reducing the receptor synthesis rate and thereby lowering the signaling bound morphogen concentration near the source end, the particular feedback mechanism (1) has a leveling effect on the (normalized) signal bound morphogen gradient $\bar{b}(x)=[L R] / \bar{R}_{0}$ which works against robustness. The manner in which the particular feedback mechanism changes the slope and convexity of the bound morphogen distribution drives the signaling gradient away from the appropriate gradient shape for the targeted tissue pattern. It would not serve the intended purpose of ensuring robustness to system characteristics changes.

The observations for the special case above were then made rigorous and extended to the general case of $n \geq 1$. For this extension, the results in Lemma 2 and monotonicity result of Proposition 7 played a key role. This reason alone requires the development of the basic existence theory for the basic model with the negative feedback (1) as presented in Section 2.

The cause of the negative results of [12] suggests more appropriate negative feedback mechanisms for mediating the sensitivity to system parameter changes and thereby ensuring robustness. Such mechanisms should not cause a substantial change in the signaling gradient shape, either by leveling the monotone decreasing gradient or steeping it and making it more convex. Some possible feedback mechanisms for this purpose are currently being investigated.

Acknowledgment: The research of F.Y.M. Wan has supported in part by NIH grants P20GM66051, R01GM067247and R01GM075309. The two NIH R01 grants were awarded through the Joint NSF/NIGMS Initiative to Support Research in the 
Area of Mathematical Biology. The research project was motivated by and has benefitted from the earlier work by the second author jointly with his UCI colleagues A.D. Lander and Qing Nie on robustness by numerical simulations.

\section{References}

[1] Amann, H., "On the existence of positive solutions of nonlinear boundary value problems," Indiana Univ. Math. J., Vol. 21, 1971, 125-146.

[2] Entchev, E.V., Schwabedissen, A. and Gonzalez-Gaitan, M., "Gradient formation of the TGSF-beta homolog Dpp," Cell Vol. 103, 2000, 981-991.

[3] Gurdon, J.B., and Bourillot, P.Y., "Morphogen gradient interpretation," Nature, Vol. 413, 2001, 797-803.

[4] Hill, A.V., "The combinations of haemoglobin with oxygen and with carbonmonoxide," I. J. Physiol., vol. 40 (iv-vii), 1910.

[5] Kerszberg, M. and Wolpert, L., Mechanisms for positional signalling by morphogen transport: a theoretical study," J. Theor. Biol., Vol. 191, 1998, 103-114.

[6] Khong, M., "Feedback and Morphogen Gradients," Ph.D. dissertation research, University of California, Irvine, in progress.

[7] Lander, A.D., Nie, Q., Vargas, B. and Wan, F.Y.M., "Aggregation of Distributed Sources in Morphogen Gradient Formation," J. Comp. Appl. Math., Vol. 190, 2006, 232-251.

[8] Lander, A.D., Nie, Q. and Wan, F.Y.M., "Do Morphogen Gradients Arise by Diffusion?" Developmental Cell, Vol. 2, 2002, 785-796.

[9] Lander, A.D., Nie, Q. and Wan, F.Y.M., "Spatially distributed morphogen production and morphogen gradient formation," Math. Biosci. Eng. (MBE), Vol. $2,2005,239-262$.

[10] Lander, A.D., Nie, Q. and Wan, F.Y.M., "Internalization and end flux in morphogen gradient formation," J. Comp. Appl. Math 2006, 232-251.

[11] Lander, A.D., Nie, Q., Wan, F.Y.M., and Xu, J., "Diffusion and Morphogen Gradient Formation - Part I: Extracellular Formulation," submitted to J. Math. Bio.

[12] Lander, A.D., Wan, F.Y.M., and Nie, Q., "Multiple Paths to Morphogen Gradient Robustness," 2005, submitted for publication. 
[13] Lou, Y., Nie, Q. and Wan, F.Y.M., "Nonlinear eigenvalue problems in the stability analysis of morphogen gradients," Studies in Appl. Math., Vol. 113, 2004, 183-215.

[14] Sattinger, D.H., "Monotone Methods in Nonlinear Elliptic and Parabolic Boundary Value Problems," Indiana University Math. J., Vol. 21, 1972, 981-1000.

[15] Smoller, J., Shock Waves and Reaction-Diffusion Equations, Springer Verlag Inc., New York, 1982.

[16] Teleman, A.A., and Cohen, S.M., "Dpp gradient formation in the Drosophila wing imaginal disc," Cell, Vol. 103, 2000, 971-980.

[17] Vargas, B., Leaky boundaries and morphogen gradient formation, Ph.D. Dissertation, University of California, Irvine, December, 2006.

[18] Lander, A.D., Q. Nie, Q., Vargas, B. and Wan, F.Y.M., "Apical-Basal Cell Depth and Morphogen Robustness," to appear.

[19] Wan, F.Y.M., Introduction to the Calculus of Variations and Its Applications, Chapman \& Hall, New York, 1995.

\section{Legends for Figures}

Figure (1): $[L R] / \bar{R}_{0}$ vs. $x$ for different combination of $\left\{\gamma, \bar{v}_{L}\right\}$ with the remaining parameters values given in Table 1 . The dash-dot curve is for $\{0,1\}$, the dash curve is for $\{0,2\}$, and solid curve is for $\{1,2\}$.

Figure (2): $\quad[L R] / \bar{R}_{0}$ for $\gamma=0$ with $\bar{v}_{L}=1$. and for $\gamma=1,2,10,50, \infty(=$ upper solution) with $\bar{v}_{L}=2$ in all five cases. All other parameter values are as given in Table 1.

\section{$9 \quad$ Figures}




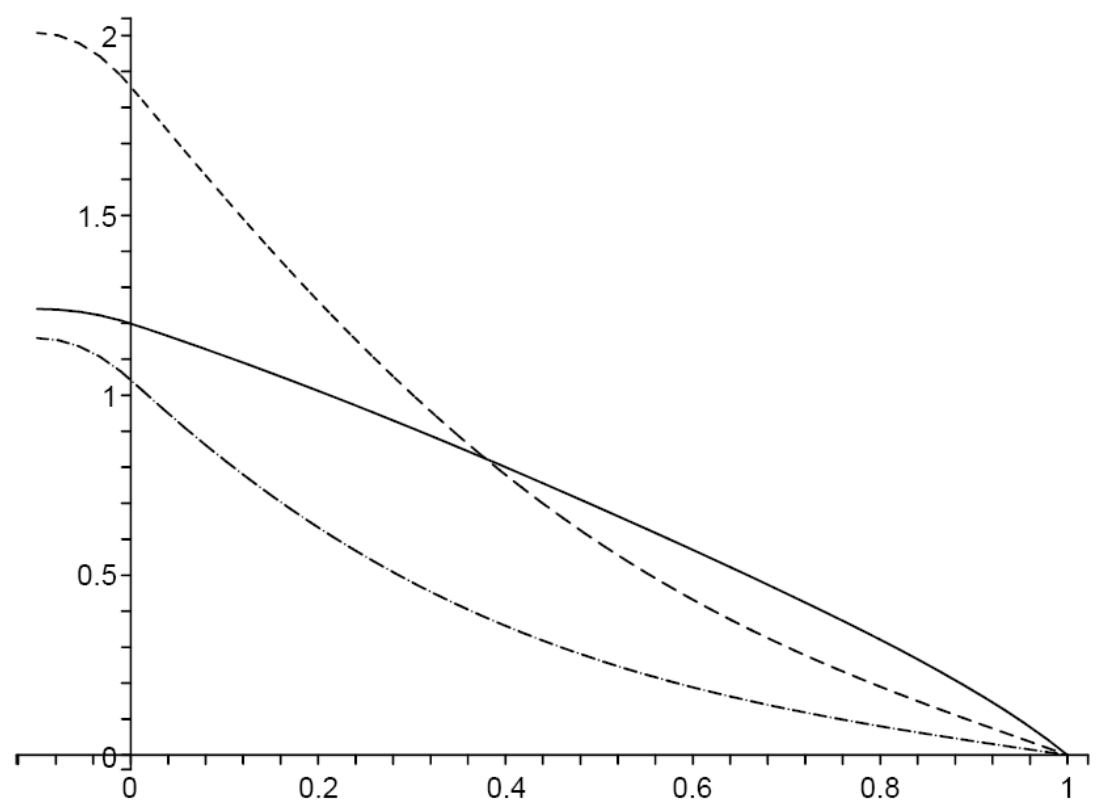

Figure 1:

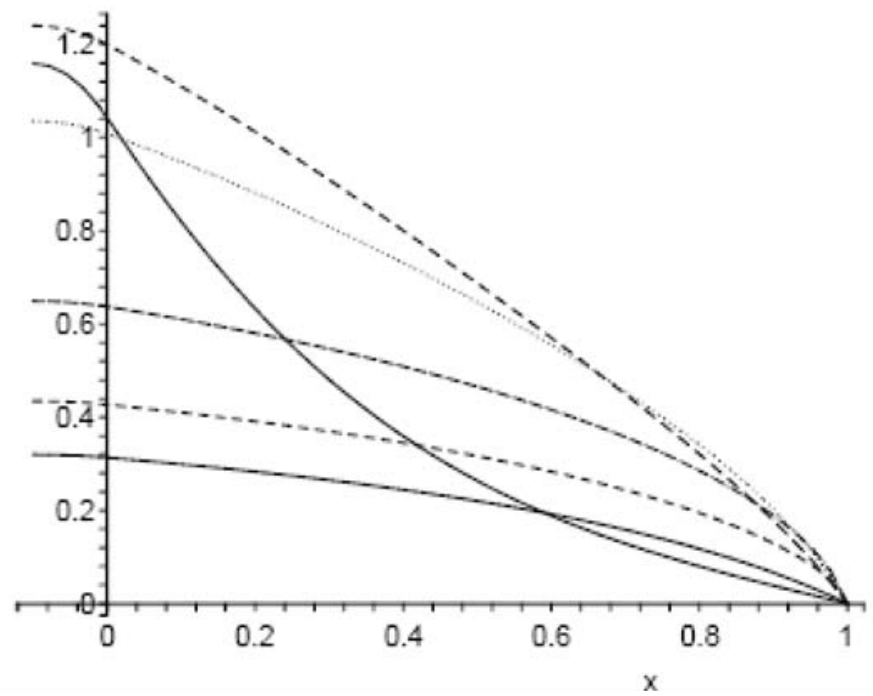

Figure 2: 